\title{
Inestabilidades de ladera provocadas por precipitaciones intensas: caso de estudio del 9 al 16 de junio de 2010 en Asturias
}

https://doi.org/10.31978/639-19-010-0.659

\author{
Manuel Antonio Mora García ${ }^{1}$ (mmoray@aemet.es) \\ María José Domínguez Cuesta² (dominguezmaria@uniovi.es) \\ Pablo Valenzuela Mendizábal ${ }^{3}$ (pablo.valenzuela.mendizabal@gmail.com)
}

${ }^{1}$ AEMET / Delegación Territorial en Castilla y León

${ }^{2}$ Universidad de Oviedo / Departamento de Geología

${ }^{3}$ Tragsatec $S$. A.

\begin{abstract}
RESUMEN
En este trabajo se presenta como caso de estudio el episodio de lluvias intensas ocurrido en junio de 2010 en Asturias, en el que, en el marco sinóptico de un patrón de bloqueo atlántico en «omega», se formaron sucesivas depresiones aisladas (cut-off low). Esta situación afectó a la península ibérica, registrándose precipitaciones intensas y persistentes durante más de una semana en el área cantábrica, las cuales dieron lugar a numerosas inestabilidades de ladera en Asturias. La descripción mesoescalar detallada de esta situación meteorológica, junto con el análisis de los datos de precipitación de la red climatológica y de los datos diarios del modelo de balance hídrico de AEMET, así como la consideración de los valores umbrales de precipitación acumulada previamente establecidos para la ocurrencia de inestabilidades en Asturias, constituyen el primer paso para poder generar mapas de riesgo que permitan establecer sistemas de alerta temprana en el futuro.
\end{abstract}

PALABRAS CLAVE: inestabilidades de ladera; landslides; precipitaciones intensas; heavy rainfall; dana; cut-off low.

\section{INTRODUCCIÓN}

Las inestabilidades de ladera o movimientos del terreno son procesos naturales que suponen la movilización de rocas, derrubios o suelos ya sea de forma repentina o dilatada en el tiempo. Su ocurrencia está relacionada con las características del terreno, que se pueden considerar factores condicionantes, y con un factor desencadenante que es el responsable último de que la inestabilidad tenga lugar.

Las inestabilidades de ladera provocan cada año numerosas víctimas y cuantiosas pérdidas económicas. En el periodo 2004-2010 se han contabilizado en el mundo 32322 víctimas humanas como consecuencia de 2620 inestabilidades de ladera no asociadas a movimientos sísmicos (PETLEY, 2012). En Latinoamérica y el Caribe, entre 2004 y 2013 hay constancia de 611 inestabilidades de ladera que causaron 11631 víctimas mortales (Sepúlveda y Petley, 2015). En Europa, las pérdidas anuales producidas por este fenómeno se estiman en unos 4700 millones de euros; además, durante el periodo 1995-2014, fallecieron al menos 1370 personas como consecuencia de 476 inestabilidades de ladera (HAQUE et al., 2016), con una distribución irregular y mayor concentración en las áreas montañosas. 
En España, entre 1995 y 2014 fallecieron 38 personas (MINISTERIO DEL INTERIOR, 2016) debido a movimientos del terreno, lo que supone el 3,2\% de fallecidos por desastres naturales. A nivel regional, en Asturias se han contabilizado 2245 inestabilidades de ladera (localmente conocidas como argayos) para el periodo 1980-2016 (VALENZUELA et al., 2017a), que han generado diversas víctimas e importantes pérdidas materiales.

Aproximadamente el $90 \%$ de las inestabilidades de ladera que produjeron víctimas a nivel global estuvieron precedidas de un periodo prolongado de precipitaciones (SASSA y CANUTTI, 2009). En el caso de la península ibérica, las precipitaciones también constituyen el principal factor desencadenante (CoROMINAS, 2005). La cantidad de lluvia acumulada y el grado de saturación del suelo, expresados generalmente en forma de umbrales, son determinantes a la hora de analizar episodios de inestabilidades de ladera en los que la precipitación es el factor desencadenante.

La protección de vidas y bienes frente a desastres naturales es una prioridad de la sociedad actual. Los avances científicos, que han supuesto un mejor conocimiento de las características geomorfológicas del terreno y una notable mejora de los modelos de predicción numérica del tiempo, han derivado en el desarrollo de los primeros sistemas de alerta temprana frente a las inestabilidades de ladera. Los inventarios de inestabilidades, las cartografías de zonas susceptibles y la determinación de umbrales de lluvia para el desencadenamiento son las herramientas fundamentales para poder establecer sistemas de alerta temprana.

Algunos países ya disponen de servicios de alerta temprana para este tipo de fenómenos, basados en precipitaciones observadas o previstas, como Japón, Noruega o Reino Unido, por lo que la contribución de los servicios meteorológicos nacionales es fundamental. Se utilizan distintos modelos o metodologías para determinar umbrales empíricos: intensidad y persistencia de las precipitaciones, redes neuronales o modelos físico-dinámicos empíricos. Además, es fundamental disponer de inventarios de movimientos del terreno lo más completos posible para poder llevar a cabo la calibración de los modelos predictivos.

A nivel regional también se utilizan otros indicadores, como la precipitación acumulada o la precipitación acumulada-duración. Sin embargo, la mayoría de los modelos no tienen en cuenta las condiciones previas de humedad del suelo, determinante para poder afinar los umbrales de precipitación.

Para el caso particular de Asturias, se han calculado distintos umbrales empíricos de precipitación acumulada en función de la humedad previa del suelo y en base a la intensidad-duración de los episodios lluviosos. En concreto, se han definido umbrales para los dos patrones meteorológicos más habituales con acumulaciones significativas de precipitación y que han resultado con un número elevado de argayos: 1) episodios lluviosos en otoño e invierno con precipitaciones moderadas y persistentes durante un periodo relativamente largo y 2) episodios de lluvias intensas durante periodos cortos, típicos de final de la primavera o comienzos del verano (VALENZUELA et al., 2017b).

En el presente trabajo se analizará el episodio de lluvias moderadas a fuertes y persistentes que tuvo lugar en la cornisa cantábrica entre el 9 y el 16 de junio de 2010, y en concreto, los aspectos relativos a las precipitaciones acumuladas y al estado de humedad del suelo.

\section{DATOS DE PRECIPITACIÓN OBSERVADOS}

Durante el periodo comprendido entre el 9 y el 16 de junio de 2010 tuvo lugar en Asturias un episodio de carácter singular en el que se registraron más de 400 mm de precipitación acumulada en algunos lugares. Se superaron los $200 \mathrm{~mm}$ en prácticamente la totalidad de las 75 estaciones de observación de AEMET en territorio asturiano, como se puede observar en la figura 1. En cuanto a las precipitaciones en 24 horas (de 07 a 07 UTC) de cada uno de los días del episodio, destacan el periodo entre los días 9 y 11 así como el día 15, como se puede apreciar en la figura 2. Los periodos de retorno calculados para precipitación acumulada en 8 días superan los 200 años (al $80 \%$ de fiabilidad) en la mayoría de los observatorios que disponen de series largas para su cálculo. Si se considera únicamente el mes de junio, el periodo de retorno supera los 1000 años, ya que el mes de junio suele ser de los más secos. 
En cuanto a la precipitación máxima recogida en 24 horas, muchas estaciones superaron los 100 mm, algo relativamente frecuente ya que los periodos de retorno calculados en base a la climatología oscilan entre 7 y 31 años, aunque, restringiéndose al mes de junio, se trata de valores anómalos.

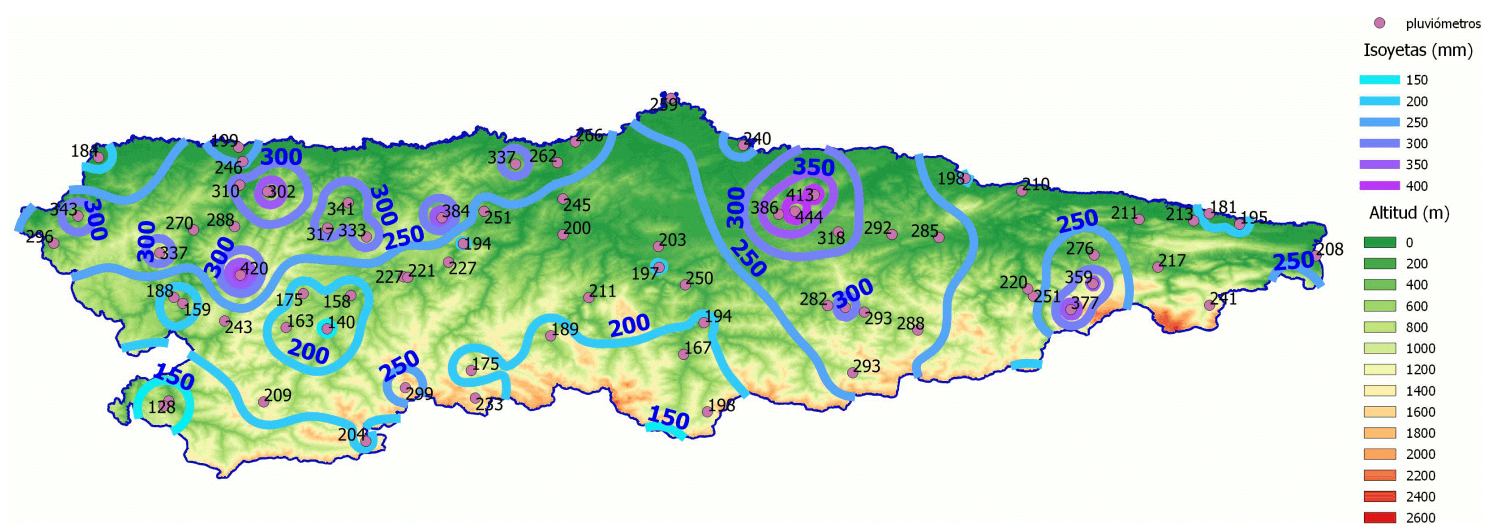

Figura 1. Precipitación acumulada (mm) entre el 9 y el 16 de junio de 2010 en Asturias en estaciones pluviométricas e isoyetas (interpolación espacial tipo kriging). Fuente: AEMET.

a)

b)

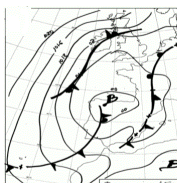

c)

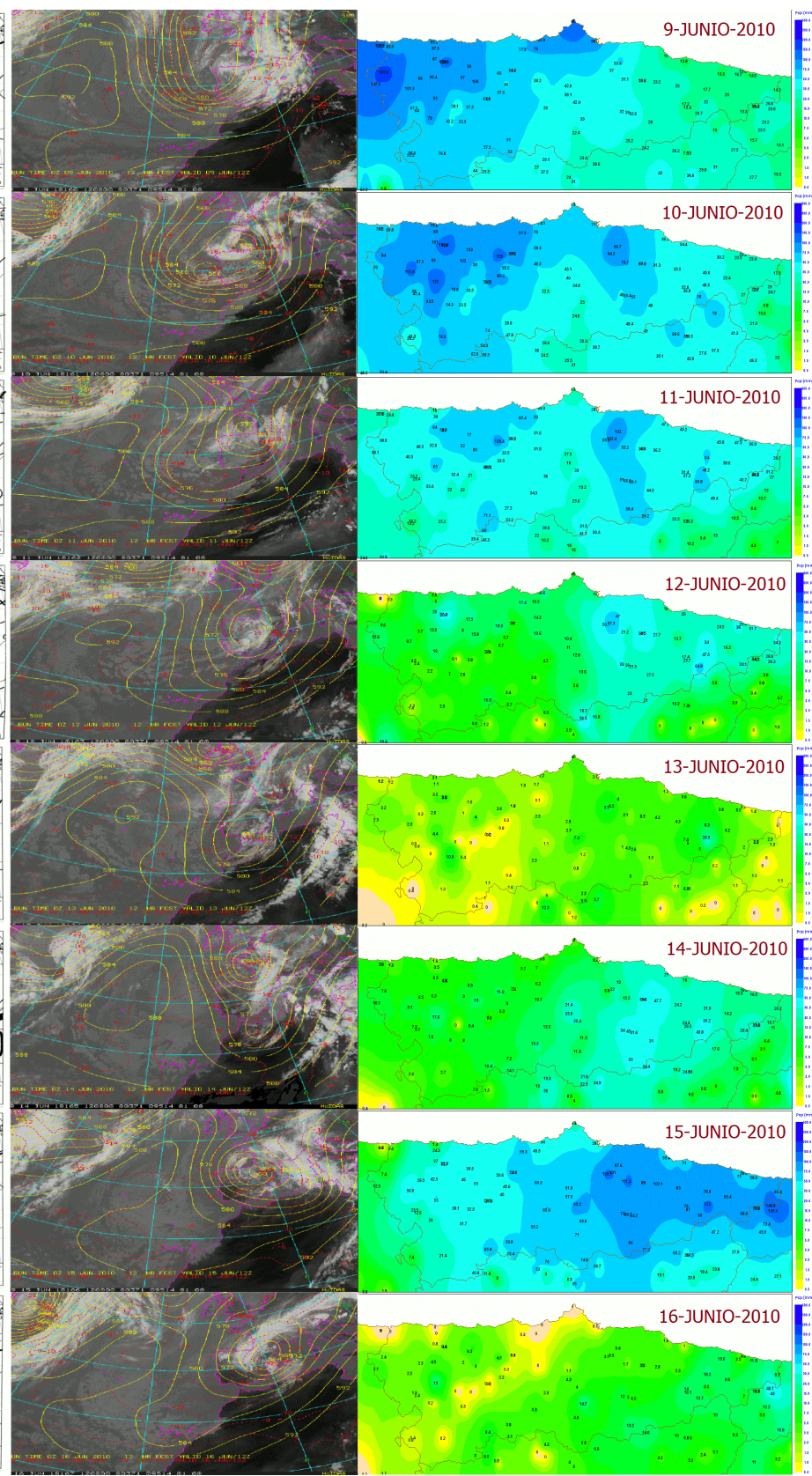

Figura 2.

De izquierda a derecha: análisis de superficie $(\mathrm{hPa})$; altura geopotencial (m), temperatura $\left({ }^{\circ} \mathrm{C}\right)$ en $500 \mathrm{hPa}$ e imagen infrarroja (12 UTC); y precipitación registrada interpolada del periodo de 7 a 7 UTC del día siguiente:

a) día 9, b) día 10, c) día 11, d) día 12, e) día 13, f) día 14, g) día 15 y h) día 16. 


\section{SITUACIÓN SINÓPTICA Y ASPECTOS MESOESCALARES}

Durante el periodo comprendido entre el 9 y 16 de junio de 2010, la configuración a escala sinóptica en el entorno de la península ibérica se caracterizó por un patrón de bloqueo atlántico en «omega», formándose sucesivas depresiones aisladas (cut-off low) que afectaron a la península ibérica, registrándose precipitaciones intensas y persistentes durante más de una semana en la cornisa cantábrica. La topografía de $300 \mathrm{hPa}$ promediada para todo el periodo refleja este patrón en «omega», con dorsal sobre las islas Azores extendiéndose hacia el norte y baja cerrada «desgajada» $\mathrm{O}$ «segregada» de la circulación general del oeste sobre el norte peninsular(figura 3a).

La anomalía respecto a la climatología fue notable, tal y como se aprecia en la figura $3 b$, en la que se pueden observar dos núcleos de anomalía de altura geopotencial muy marcados: positivo en el Atlántico norte y negativo en la península ibérica.

a)

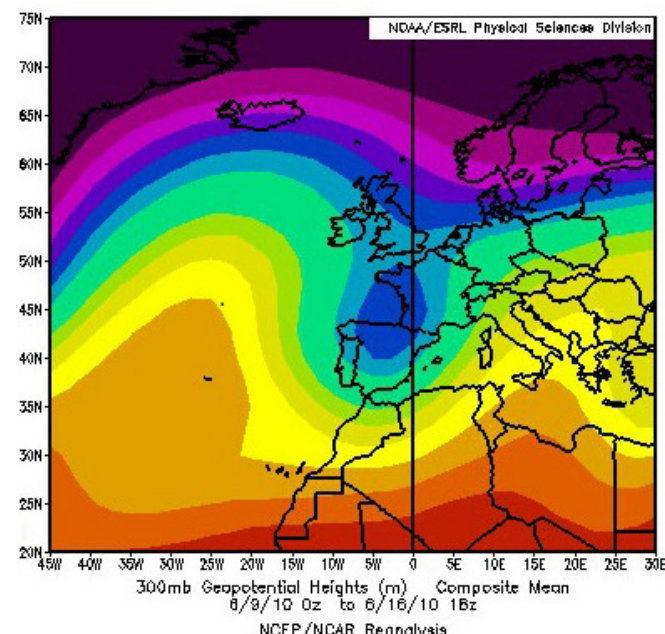

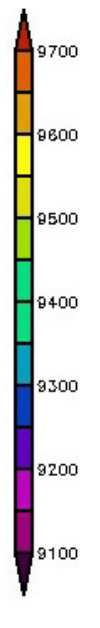

b)

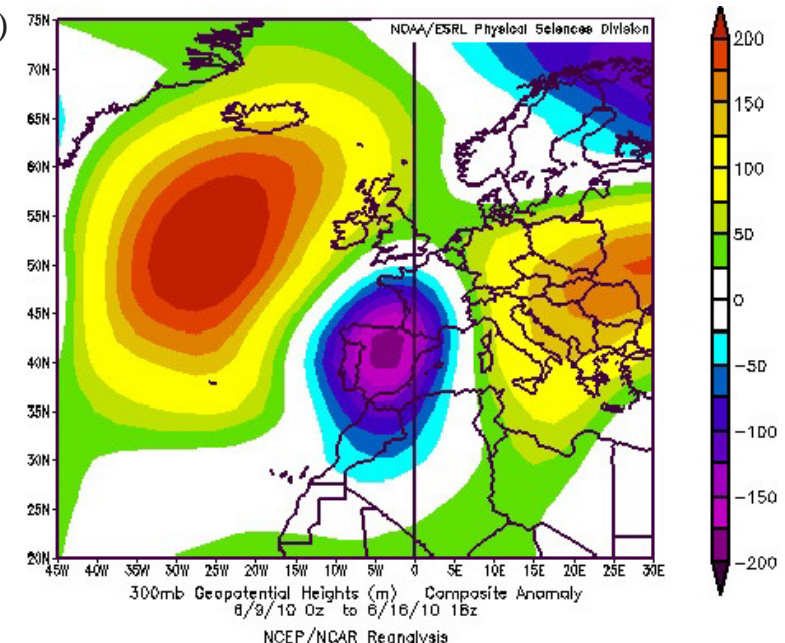

Figura 3. a) Reanálisis (NCEP/NCAR) promediado de geopotencial en $300 \mathrm{hPa}$ entre los días 9 y 16 de junio de 2010. b) Anomalía respecto a la climatología de geopotencial en $300 \mathrm{hPa}$ del mismo periodo.

Fuente (NOAA/ESRL).

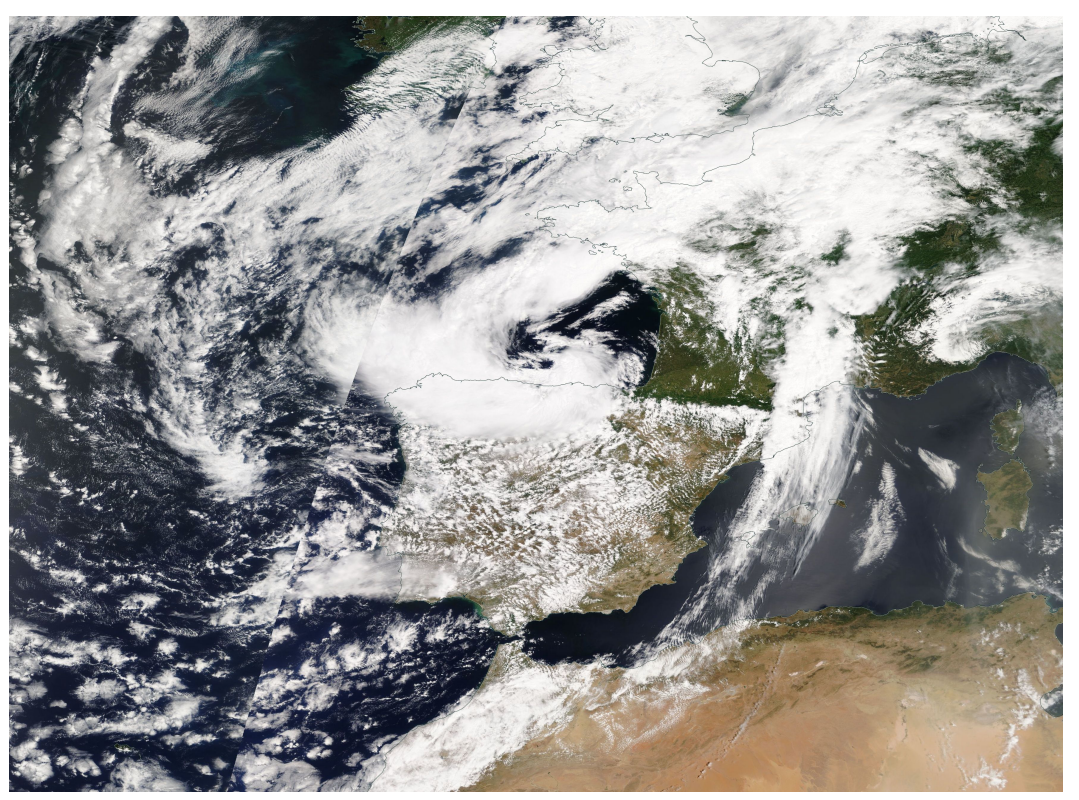

Figura 4. Composición imágenes sensor visible MODIS de los satélites de órbita polar de la NASA.

Día 10 de junio de 2010. 
A escala mesobeta es de destacar la importancia de la ubicación del centro de la baja en superficie y el flujo marítimo sobre Asturias en conjunción con la persistencia del frente ocluido, factores determinantes para la persistencia de las precipitaciones. En ese sentido es reseñable que el frente ocluido asociado a la baja, permaneció muy activo durante el proceso de oclusión (Schultz y VAughan, 2011). En la figura 4, la composición de imágenes del sensor visible MODIS, muestra cómo el frente ocluido afecta al área cantábrica enroscado en espiral alrededor de la baja en superficie durante el día 10.

\subsection{Descripción cronológica del episodio}

A continuación se describe, por orden cronológico, la evolución de las principales estructuras sinópticas presentes durante el episodio entre los días 9 y 16 de junio de 2010 (figura 2).

- Día 9. La vaguada en altura, con embolsamiento de $-18^{\circ} \mathrm{C}$ en $500 \mathrm{hPa}$, afecta al noroeste peninsular; el forzamiento dinámico y térmico da lugar a precipitaciones ocasionalmente tormentosas más significativas en la mitad occidental, mientras que el frente asociado a la baja en superficie comienza el proceso de oclusión.

- Día 10. La baja en superficie se ubica en el Cantábrico, frente a las costas asturianas, mientras, en niveles medios, la baja perfectamente aislada presenta dos núcleos fríos. El frente ocluido y el flujo marítimo del norte originan precipitaciones notables en buena parte del territorio asturiano.

- Día 11. La baja fría se desplaza hacia el golfo de Vizcaya; el flujo marítimo del norte y el frente ocluido que permanece activo siguen originando precipitaciones notables sobre Asturias.

- Día 12. La baja en niveles medios y altos, que mantiene un núcleo frío de $-18^{\circ} \mathrm{C}$ en $500 \mathrm{hPa}$, se desplaza rápidamente hacia el sur. En niveles bajos persiste el flujo del norte y el frente ocluido, ya debilitado, continúa originando precipitaciones localmente moderadas en Asturias.

- Día 13. Jornada de transición, con precipitaciones débiles en la región asturiana, asociadas al flujo marítimo; la baja en niveles medios y altos sigue desplazándose hacia el sur mientras que por el norte, en latitudes altas, comienza a descolgarse una dana (depresión aislada en niveles altos).

- Día 14. La depresión aislada, con núcleo frío de $-24{ }^{\circ} \mathrm{C}$ en $500 \mathrm{hPa}$, se ubica en la Bretaña francesa, persistiendo el flujo del norte sobre el área cantábrica que da lugar a precipitaciones débiles, localmente moderadas en los Picos de Europa.

- Día 15. La dana se centra en el noreste peninsular, con un núcleo frío de $-22{ }^{\circ} \mathrm{C}$ en $500 \mathrm{hPa}$. El forzamiento dinámico y térmico da lugar a precipitaciones ocasionalmente tormentosas, más significativas en la mitad oriental. Se observan las características clásicas de la dana, convección del núcleo frío y banda baroclina que se representa como un frente ocluido en el mapa de superficie.

- Día 16. La baja fría, ya con reflejo en superficie, se desplaza hacia el este. Aunque el frente ocluido sigue originando precipitaciones moderadas, sobre todo en el País Vasco, en la región asturiana son de carácter más débil, asociadas al flujo del norte en superficie.

\section{IMPACTOS}

Estas precipitaciones intensas y persistentes sobre Asturias originaron inundaciones, fundamentalmente por el desbordamiento de los cauces fluviales. Destacan los desbordamientos de ríos como el Nalón, o el Sella y el Piloña que obligaron a intervenir a la Unidad Militar de Emergencias para evacuar a los pacientes del hospital de Arriondas, al quedar esta localidad prácticamente aislada. Además de numerosas carreteras cortadas, se interrumpieron las líneas férreas que unen Asturias con Cantabria y con la Meseta. Se contabilizaron tres 
fallecidos, más de 300 personas evacuadas y se concedieron ayudas por valor de 60 millones de euros con objeto de paliar los daños materiales.

Una vez alcanzada la saturación de los suelos, las lluvias posteriores dieron lugar a numerosas inestabilidades de ladera en Asturias. Se tiene constancia documentada de la ocurrencia de 164 argayos entre el 1 de mayo y el 30 de junio de 2010, aunque únicamente se dispone de información precisa del momento de ocurrencia de 95 de ellos (figura 5). Estos 95 eventos se han tomado como base para la determinación de los umbrales de lluvia que desencadenaron inestabilidades de ladera asociados a este episodio.

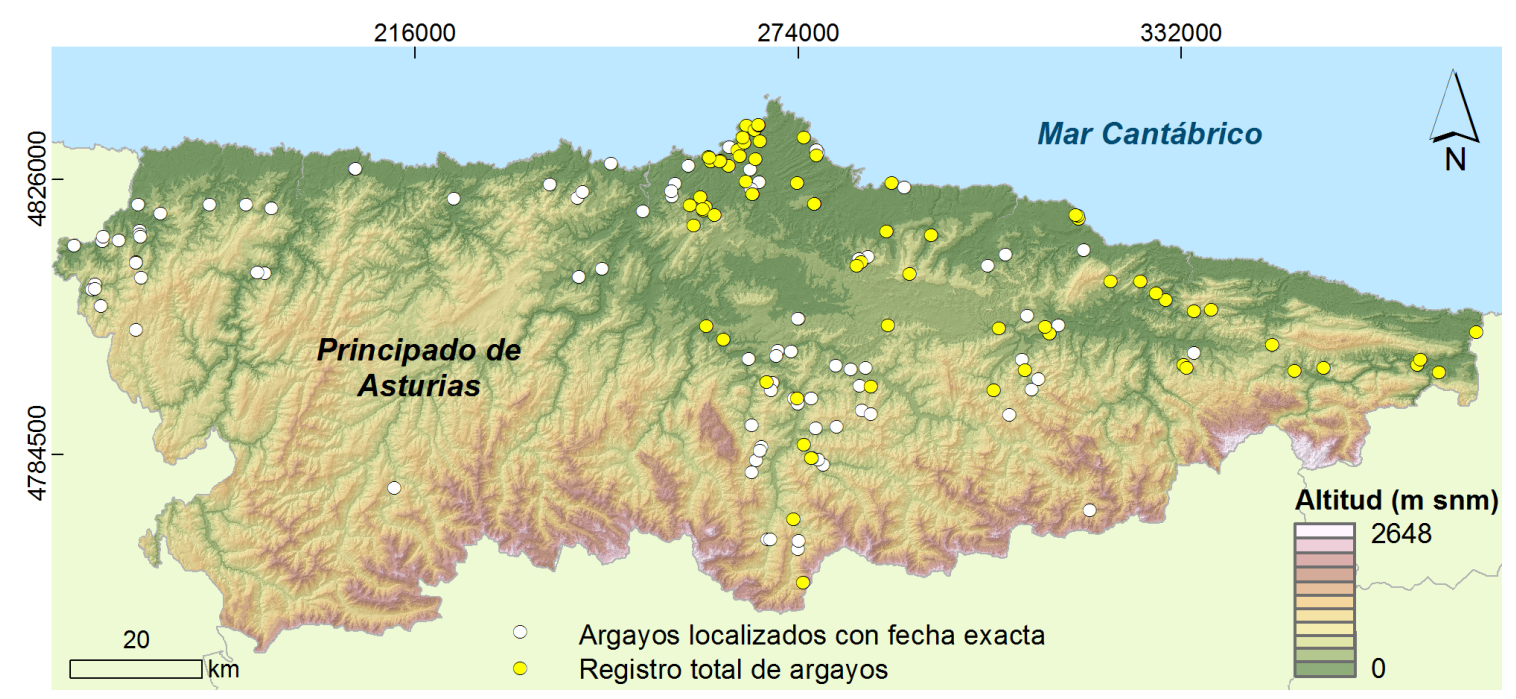

Figura 5. Inestabilidades de ladera producidas durante el episodio de junio de 2010.

\section{BALANCE HÍDRICO, UMBRALES Y PATRONES DE PRECIPITACIÓN}

Estudios preliminares (VALENZUELA et al., 2017b) sobre este mismo caso de estudio señalan que el $98 \%$ de las inestabilidades de ladera que se desencadenaron durante este episodio tuvieron lugar con porcentajes de agua útil en el suelo del 99-100 \%. Una vez saturado el suelo, la cantidad de precipitación acumulada para el desencadenamiento de cada uno de los distintos argayos ocurridos oscila entre 110 y $330 \mathrm{~mm}$. A partir de los datos disponibles, asociando a cada argayo un pluviómetro de referencia próximo o el dato de precipitación interpolado, se calcularon distintos umbrales intensidad-duración, que definen las condiciones de lluvia que deben excederse para que ocurra una inestabilidad. Como complemento al establecimiento de umbrales, también se han analizado las situaciones sinópticas que dan lugar a este tipo de precipitaciones con el resultado del desencadenamiento de inestabilidades de ladera en Asturias (VALENZUELA et al., 2018), distinguiéndose cuatro patrones sinópticos, uno de los cuales corresponde al caso analizado (cut-offlow).

\section{CONCLUSIONES}

Las precipitaciones asociadas a este episodio en Asturias fueron significativas y su carácter persistente se debió a un patrón sinóptico de bloqueo atlántico en «omega». Las sucesivas depresiones aisladas que se descolgaron de latitudes altas determinaron el marco mesoescalar donde el papel desempeñado por los frentes ocluidos activos fue determinante en la distribución final de las precipitaciones. Entre los impactos de este tipo de episodios de precipitaciones significativas y persistentes se encuentran las inestabilidades de ladera. 
La disponibilidad de modelos de predicción numérica de alta resolución, así como de modelos de balance hídrico, junto con el actual estado de conocimiento sobre la ocurrencia de inestabilidades de ladera en Asturias, permite afrontar la elaboración de mapas de peligrosidad. Con ello, se podría abordar, de forma experimental, la creación automatizada de mapas de riesgo ante este tipo de eventos, estableciendo diferentes niveles que podrían ser de utilidad para los servicios de protección civil en la implementación de sistemas de alerta temprana que contribuyesen a salvaguardar vidas y bienes humanos.

\section{AGRADECIMIENTOS}

A Pilar Sanz, de la Delegación Territorial de AEMET en Cantabria, por su amabilidad y asesoramiento. A Inmaculada Abia y Diego Cubero, de la Delegación Territorial de AEMET en Castilla y León por su ayuda en la elaboración de los gráficos.

\section{REFERENCIAS}

Corominas, J., 2005. Impacto sobre los riesgos naturales de origen climático: inestabilidad de laderas. En: Moreno, J. M. (coordinador). Proyecto ECCE. Evaluación Preliminar de los impactos en España por efecto del Cambio Climático. Madrid, Ministerio de Medio Ambiente. pp. 549-579.

FuKUOKA, M., 1980. Landslides associated with rainfall. Geotechnical Engineering, 11, 1-29.

Haque, U., Blum, P., da Silva, P., Andersen, P., Pilz, J., Chalov, S., Malet, J., Aufliè, M., Andrés, N., Poyiadi, E., Lamas, P., Zhang, W., Peshevski, I., Pétursson, H., Kurt, T., Dobrev, N., García-Davalillo, J., Halkia, M., Ferri, S., Gaprindashvili, G., Engström, J., Keellingset, D., 2016. Fatal landslides in Europe. Landslides, 13, 1545-1554.

Ministerio del InTERIOR, 2016. Anuario Estadístico del Ministerio del Interior 2014. Ministerio del Interior. Secretaria General Técnica. Gobierno de España.

PeTLEy, D., 2012. Global patterns of loss of life from landslides. Geology, 40 (10), 927-930.

SepúlvedA, S. y Petley, D., 2015. Regional trends and controlling factors of fatal landslides in Latin America and the Caribbean. Nat. Hazards Earth Syst. Sci., 15, 1821-1833.

Sassa, K. y CanutTi, P., 2009. Landslides disaster risk reduction. Springer, Berlin Heidelberg.

Schultz, D. y Vaughan, G., 2011. Occluded fronts and the Occlusion process A Fresh Look at Conventional Wisdom. Bull. Amer. Meteor. Soc., 92, 443-466.

Valenzuela, P., Domínguez-Cuesta, M. J., Mora, M., 2017a. A spatio-temporal landslide inventory for the NW of Spain: BAPA database. Geomorphology, 293A, 11-23.

Valenzuela, P., Domínguez-Cuesta, M. J., Mora, M., JimÉnez-SÁnchez, M., 2017b. Rainfall thresholds for the triggering of landslides considering previous soil moisture conditions (Asturias, NW Spain). Landslides, $15,273$.

Valenzuela, P., Iglesias, M., Domínguez-Cuesta, M. J., Mora, M., 2018. Meteorological Patterns Linked to Landslide Triggering in Asturias (NW Spain): A Preliminary Analysis. Geosciences, 8 (1), 18. 
